\title{
VLT Interferometry in the Data Flow System
}

\author{
P. Ballester ${ }^{1 \mathrm{a}}$, T.Licha ${ }^{\mathrm{b}}$, D.J.McKay ${ }^{\mathrm{a}, \mathrm{c}}$, I. Percheron $^{\mathrm{a}}$, \\ M. Peron ${ }^{a}$, A. Richichi ${ }^{a}$, C. Sabet ${ }^{b}$, M. Wittkowski ${ }^{a}$ \\ ${ }^{a}$ European Southern Observatory, Karl-Schwarzschildstr. 2, D-85748 Garching, Germany; \\ ${ }^{\mathrm{b}}$ Tekom GmbH, Gautinger Str. 29, D-82152 Krailing, Germany \\ ${ }^{\mathrm{c}}$ Rutherford Appleton Laboratory, Didcot, OX11 0QX, United Kingdom
}

\begin{abstract}
Science interferometry instruments are now available at the Very Large Telescope for observations in service mode; the MID-Infrared interferometry instrument, MIDI, started commissioning and has been opened to observations in 2003 and the AMBER 3-beam instrument shall follow in 2004. The Data Flow System is the VLT end-to-end software system for handling astronomical observations from the initial observation proposal phase through to the acquisition, archiving, processing, and control of the astronomical data. In this paper we present the interferometry specific components of the Data Flow System and the software tools which are used for the VLTI.
\end{abstract}

Keywords: Very Large Telescope, Interferometer, Data Flow System, Data Handling

\section{INTRODUCTION}

Since obtaining the first VLTI fringes, three years ago, several sub-systems have been installed at Paranal Observatory to support science instruments ${ }^{1}$. A first scientific instrument for MID-infrared beam recombination, MIDI, started commissioning in 2003 and is now offered to the community for science operations. A second instrument, the Astronomical Multi-BEam Recombiner, AMBER, that supports three-beams, near-infrared combination, is currently in the phase of commissioning. Additional systems will be added to the interferometer that will expand its observational capabilities. This includes a set of four repositionable, $1.8 \mathrm{~m}$ diameter, Auxiliary Telescopes and a dual-feed system for astrometry measurements, PRIMA.

The VLTI instruments are engineered, commissioned, and operated like other VLT instruments. Science instruments like MIDI that are already offered for observations can be used for visitor-mode as well as service-mode observation programs. In visitor mode, the astronomer is present at the telescope and can adapt the observation program to specific target properties, changing observing conditions, or calibration needs. In service-mode ${ }^{4}$ the observatory scientific operators perform the observations and the data are processed and sent to the requesting astronomer. Most observations in interferometry will involve measurements for different spatial frequencies, spread over periods of several weeks and are likely to require different configurations. The service mode observation is therefore expected to be a dominant mode of the VLTI.

Conducting observations in service mode requires the Data Flow System ${ }^{3}$ for handling astronomical observations from the initial observation proposal phase through to the acquisition, processing and control of the astronomical data. The procedure for proposal preparation in the Data Flow System involves a two-stage proposal preparation process: Phase 1 and Phase 2. In Phase 1, proposals are submitted electronically to ESO and evaluated by the Observing Program Committee (OPC). After the OPC selection has taken place and a schedule has been created, Phase 2 preparation is then carried out that involves the creation of Observation Blocks. Each Observation Block specifies an individual observation sequence which can be scheduled and executed completely without interruption. Upon execution of the OBs, the produced data are first stored in an on-line archive system at Paranal observatory and then in the VLT archive

pballest@eso.org; phone +49 8932006 435; fax +49 8932023 62; http://www.eso.org; European Southern Observatory, KarlSchwarzschildstr. 2, D-85748 Garching, Germany 
in Garching. The Science Archive stores all raw frames produced by the instruments as well as reference calibration data and $\log$ files including maintenance and ambient conditions logs. The Science Archive is available to archive researchers and astronomers for catalog access and retrieval of scientific data as they become available at the end of the proprietary period as well as retrieval of calibration data as soon as they have been processed and verified by the Data Flow Operations. An on-line pipeline infrastructure is available at the telescope for immediate processing and quality assessment of the data. In Service mode the data are processed off-line in Garching before being controlled by a Quality Control scientist and subsequently packaged for the user. This science operations process is described from the user's point of view in [12], [7] describes the preparation of MIDI science operations, and [9] presents the current status of interferometry quality control.

In this paper we present the interferometry specific software components of the Data Flow System and the software tools for the VLTI instruments VINCI and MIDI. With each new VLTI instrument, a milestone for the accuracy of the angular measurements will be reached: For MIDI it is about 20 milliarcsec at 10 microns. For AMBER it is 1 milliarcsec and later, for PRIMA, which will provide high-accuracy astrometry measurements, the ultimate goal of 10 microarcsec is expected. Extracting the science information from these measurements requires a thorough understanding of the data obtained by modeling, homogeneous calibration of large datasets and robust data reduction methods.

\section{VERY LARGE TELESCOPE INTERFEROMETER}

The VLT Interferometer consists of an infrastructure ${ }^{1}$ including delay lines, fringe tracking, and adaptive optics systems and of instrumentation. Commissioning of the VLTI delay lines has been performed with a specific beam combiner instrument, VINCI. The latter is based on the proven concept of FLUOR (Fiber Linked Unit for Optical Recombination) which has been operated since 1995 as a focal instrument of the IOTA interferometer in Arizona. VINCI has been used for commissioning observations with siderostat telescopes $(40 \mathrm{~cm}$ diameter) and then with the VLT Unit Telescopes.

The VLT Interferometer provides 40 stations with baselines ranging from 16 to $202 \mathrm{~m}$. The complete set of array configurations is available to the Auxiliary Telescopes, repositionable $1.8 \mathrm{~m}$ telescopes, that are $100 \%$ dedicated to VLTI and will enable full time use of the VLTI facilities. The scientific instrumentation plan of the VLTI includes three facilities: MIDI, AMBER and PRIMA.

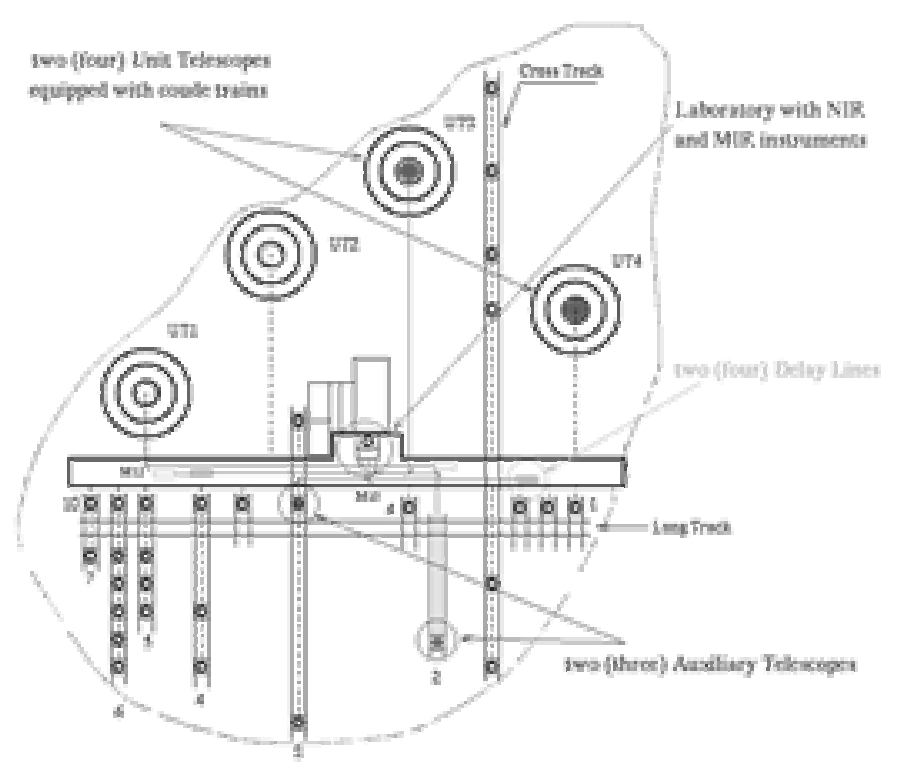

Fig. 1: VLTI platform diagram with observation stations for the Auxiliary Telescopes.

MIDI $^{5}$ covers the wavelength range 8 to 12 microns and records spectrally-dispersed fringes, making it possible to reach a resolution $(\lambda / B)$ of 20 milliarcsec at 10 microns. The main scientific objectives include the study of protostars, very 
young stars, circumstellar discs, brown dwarfs, tori around galactic nuclei, the centre of our own galaxy and the search for exoplanets. Recent observations of the Seyfert core of NGC1068 in the near-infrared ${ }^{11}$ (using the VINCI instrument) as well as in the mid-infrared ${ }^{2}$ (MIDI) illustrate the possibilities offered by infrared long-baseline interferometry ${ }^{10}$. MIDI started commissioning at the VLTI in 2003 and is now offered for operations since the beginning of the current VLT observing period P73. With MIDI, an interferometer is offered to the whole community for the first time.

AMBER which combines up to three beams is the first VLTI instrument with some imaging capability. It delivers spectrally dispersed fringes covering the three near-infrared bands J, H, K. Three spectral resolutions of approximately $35,1000,10000$ are supported. By analysing three beams at once it is possible to obtain images through phase closure techniques eliminating the influence of atmospheric turbulence on fringe position. The system can also be used in differential interferometry mode in order to estimate the phase difference between two spectral channels. The wavelength coverage will be extended in a second phase down to $0.6 \mu \mathrm{m}$ at the time the ATs become operational. The magnitude limit of AMBER is expected to reach $\mathrm{K} \cong 20$ when a bright reference star is available and $\mathrm{K} \cong 14$ otherwise. The main scientific objectives are the investigation, at very high angular resolution, of disks and jets around young stellar objects, active galactic nuclei dust tori, the search for exoplanets, the study of stellar properties such as diameter, pulsation, or mass loss.

The objective of PRIMA is to enable simultaneous interferometric observations of two objects - each with a maximum size of 2 arcsec - that are separated by up to 1 arcmin, without requiring a large continuous field of view. PRIMA can be subdivided into five sub-systems that are positioned in different locations of the VLTI. It is composed of a star separator that feeds two arbitrary objects into the Delay Lines, a laser metrology system to monitor the internal OPD between object and reference star, a differential Delay Line to adjust the OPD between object and reference star, a fringe sensor unit and an astrometry detector allowing the observation of the fringe patterns of both stars on the same detector.

\section{VLTI DATA FLOW MODELLING TOOLS}

At the VLTI, observation preparation would be practically impossible without modeling tools allowing the user to take into account the different constraints on the observability of the target. Two applications are provided for this purpose. The first tool is the VLTI Visibility Calculator, VisCalc, which makes it possible to evaluate the observability and to compute wavelength dependent visibilities on a given target. The second tool is the VLTI Calibrators Selection tool, CalVin, which allows the user to search the ESO database of interferometry calibrators. These models involve the following components:

- Target Spectrum and Geometry: The declination and spectral energy distribution, as well as the source geometry, are parameters used to specify the observation target. Visibilities are calculated analytically for uniform discs, gaussian discs and binaries.

- User-Provided Target Models: Visibilities may also be calculated numerically for a user-provided brightness distribution which is uploaded as a FITS file.

- Observability and shadowing constraints: The user-specified observation conditions include the starting hour angle and the duration of the observation. A 3-dimensional model of the VLTI platform is used to estimate the shadowing of the auxiliary telescopes by the VLT Unit Telescopes.

- Array Configuration: a table of station positions as well as a calculation of the Optical Path Difference is used for the estimation of the projected baseline

- Instrument Configuration: transmission and dispersion models of the instrument are used for the calculation of dispersed visibilities.

VisCalc provides calculations of simulated dispersed visibilities based on software models of the VLTI instruments.

Different results can be displayed (Fig. 2) including the uv-tracks, the input image and its Fourier transform, plots of visibility versus time, visibility squared versus time, loss of correlated magnitude or the illumination distribution.

The calibrator selection tool (CalVin) provides a similar interface and involves a two stage selection process. In the first stage, the target coordinates, the array configuration and the instrument configuration can be selected. In the second stage, the default search criteria are displayed on an intermediate page and can be refined. As shown in Fig. 2, the 
results page provides the table of matching calibrators. For all matching calibrators, the visibility and observability information is calculated and displayed. It is then possible to use VisCalc for a more comprehensive calculation of the visibility information.

Both tools can be accessed from the VLT Exposure Time Calculators page on http://www.eso.org/observing/etc. The standard version shows only those configurations that are offered for the current Call for Proposals. It is updated for each new Call for Proposals in order to reflect the offered VLTI baseline configurations and instrument modes. An "expert" version, accessible from the ETC preview page (http://www.eso.org/observing/etc/preview.html) offers an extended interface with many more choices. It supports the modes and configurations that are currently not offered.

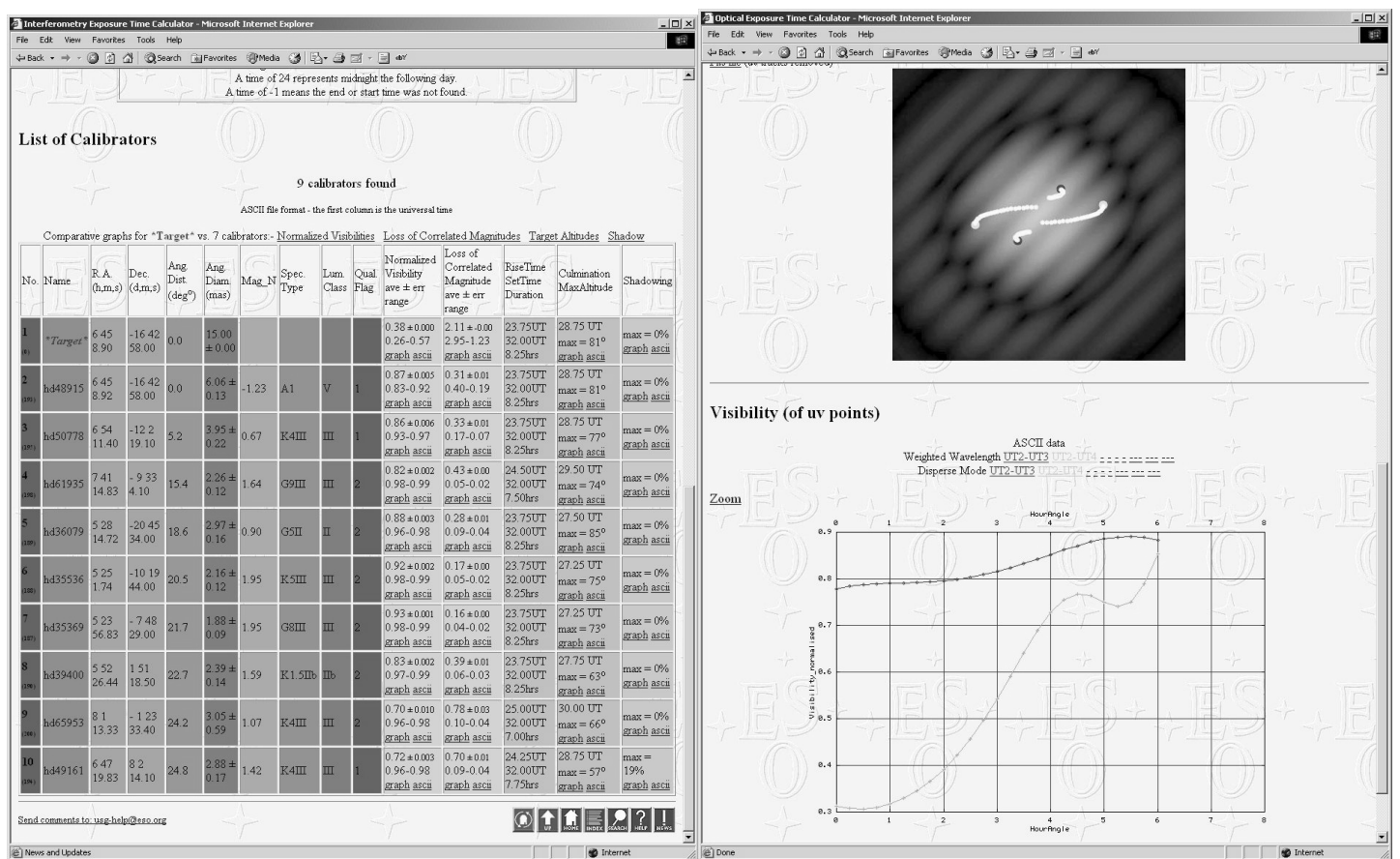

Fig. 2: Sample results from the calibrator selection tool CalVin (left) and from the visibility calculator VisCalc (right).

Exposure time calculators are also being prepared for the VLTI instruments ${ }^{6}$. These models help to understand the performance of the VLTI observation instruments over the two-dimensional parameter space of brightness and visibility that needs to be assessed in interferometry. The models are being compared to the large data sets, obtained with the available VINCI and MIDI instruments, uniformly processed data with the VLTI pipelines. Calibration and performance model fitting in interferometry often requires the homogeneous pipeline reduction of large collections of observations covering several years of observation.

\section{VLTI DATA REDUCTION PIPELINES}

The on-line pipeline infrastructure at Paranal provides an environment where the pipeline recipes are executed automatically. This task includes the recognition of the type of raw frames (an input data file with classification information), assignment of the appropriate reduction recipe, selection of the appropriate reference data and the execution of the reduction steps. The pipeline infrastructure receives the raw frames from the on-line archive system. It then classifies the incoming frames in accordance with a set of rules. 


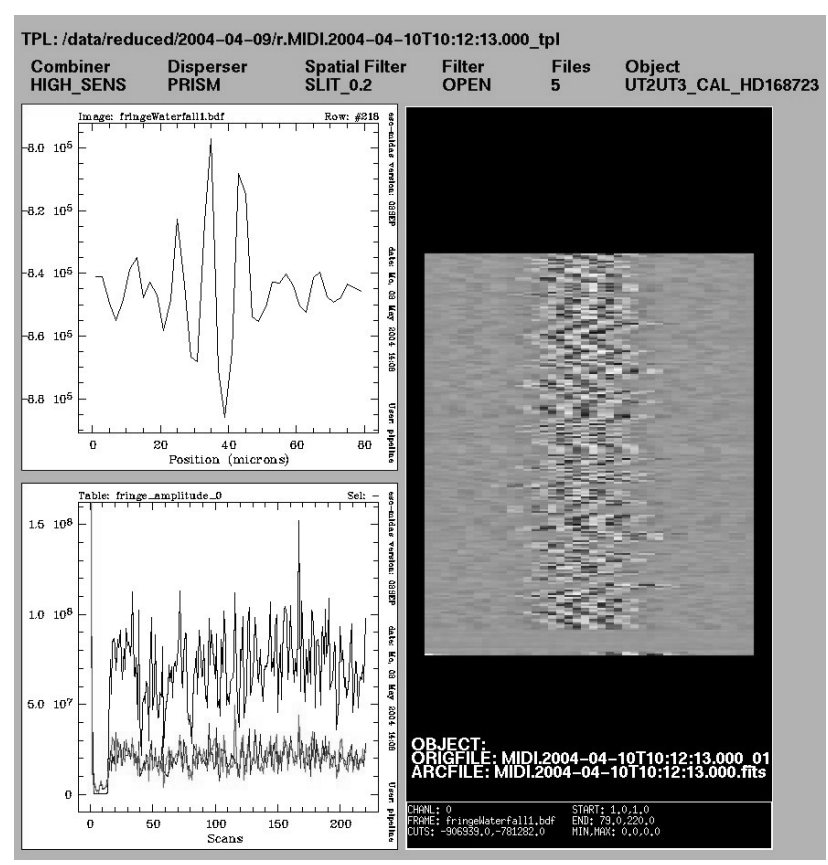

Fig. 3: MIDI waterfall display. This example of the output display illustrates three components. The image on the righthand-side is the so-called 'waterfall display' which shows the fringes in the entire data. The diagram on the top lefthand corner displays the central fringe and the one on the bottom left-hand corner is the result of the fringe amplitudes for the entire scan.

The MIDI raw data are delivered to the pipeline in the form of several FITS files. These files contain the results of both interferometry as well as the photometry observations, for calibrators and science targets. Since the interferometry data volume is rather large (about 1 Gbyte per visibility point) it is delivered in several split FITS files. The procedure for the data reduction involves two stages. In the first stage, when calibration data arrives, the raw undispersed visibilities are calculated from the dispersed-fringe data (Fig. 3). Additionally, the theoretical visibilities are also computed. This is achieved by extracting the necessary parameters from the calibrator database, based on the same list as used by the webbased calibrator selection tool CalVin. From the calibrator raw visibilities and the theoretical visibilities, the transfer functions are computed. In the second stage, when the science data is available, the calibrated visibilities are computed by using the above transfer function as well as the raw undispersed science visibilities. At Paranal, the Instrumental Transfer function can only be derived from observations that immediately precede that of the science object. In Garching, the association process can make use of all the calibration data taken during a certain period of time, including calibrations taken after the science observation. It is possible for instance to use either the closest calibrator in time or any other more suitable calibrators.

With MIDI, one had to face the challenge of large data volume (20 Gbyte per night for MIDI) and therefore all efforts have been made to streamline the processing. The pipeline recipes currently make it possible to process one night of data (about 20 Gbytes) in about half an hour. This enables immediate on-line data quality assessment and provides ample time for the off-line data quality control at Garching ${ }^{6}$. With PRIMA, homogeneous calibration will be even more important (see Section 6).

The AMBER pipeline recipes have to extract 3 visibility measurements entangled into the interferometry signal. A cornerstone of this reduction procedure is the calibration of the pixel-to-visibility matrix (P2VM). After basic detector corrections are applied, including bad pixel map and internal detector flat-field correction, an internal calibration procedure provides the P2VM for the current instrument setup. The P2VM calibration is applied to the science observation to yield the visibility measurements on the different baselines. 
The off-line processing at Garching yields a number of product files providing the visibility results and all the information for the Quality Control. This allows the QC scientist and the PI to quickly assess the quality of the data. All observations are processed with a set of pre-defined parameters, which are a compromise to allow both consistent quality control and science reductions. In some cases, the resulting science products alone (e.g. uncalibrated visibilities with uncertainties) may be sufficient to achieve the overall science objectives. However, it is usually expected that some data re-processing will be necessary to achieve the optimal result.

\section{INTERFEROMETRY DATA AND QUALITY CONTROL}

In interferometry a large effort has been made to provide a coherent description of the raw instrument data as well as the processed data in order to promote the emergence of common analysis tools and to facilitate the comparison of measurements obtained with different interferometers.

Interferometry data are usually stored in the form of FITS binary tables. This is because in most cases only a fraction of the detector is used to read the data. Several discussions are still taking place in the interferometry community for the definition of a general data structure within the FITS standard ${ }^{8}$. The header of the data follows the ESO Data Interface Control guidelines and provides the FITS keywords necessary for the data handling, processing, archive retrieval and proper documentation of the data. The binary tables of the VLTI Interferometry data are cross-referenced. The ARRAY_DESCRIPTION table provides the description and bibliographical references to the indexes used in the ARRAY_GEOMETRY and OPTICAL_TRAIN tables. The INS_DESCRIPTION table provides the description and bibliographical references to the indexes used in the INS_TRAIN table. The ARRAY_GEOMETRY table describes the geographical information about the interferometer array. The OPTICAL_TRAIN table describes the configuration of the VLTI optical elements up to the instrument entrance window. The INS_TRAIN table describes the configuration of the instrument optical elements. The IMAGING_DETECTOR describes the detector settings and the IMAGING_DATA table contains the detector data.

The product FITS files are written in accordance with the IAU Exchange format and standard ${ }^{8}$. These FITS files have the following extensions:

- Primary: consists only of a header.

- OI_ARRAY: consists of a header and a binary table. This extension describes the parameters of the telescopes.

- OI_TARGET: consists of a header and a binary table. This extension describes the parameters of the target.

- OI_WAVELENGTH: consists of a header and a binary table. This extension describes the wavelength parameters

- OI_VIS: consists of a header and a binary table. This extension describes all the parameters related to the visibility amplitudes

- OI_VIS2: consists of a header and a binary table. This extension describes all the parameters related to the visibility squared.

Quality Control at Garching consists mainly of verifying the quality of the data, producing calibration data and monitoring the instruments on different time scales. During operation, several types of calibration data are evaluated for the purpose of quality control. These include technical calibrations such as the determination of the read out noise, the characterisation of the detector linearity, the stability of the reference pixels, the dispersive elements transmission, the wavelength calibration and the determination of the output of the instrument using a known source, and the calibration of the science target using an astronomical calibrator. The technical calibrations are used for the scientific data reduction as well as for the monitoring of the health of the instrument in order to detect any problems (for example transmission degradation of an optical element). More information on Quality Control can be found on www.eso.org/observing/dfo/quality. 


\section{DATA FLOW SYSTEM FOR PRIMA}

PRIMA will be the phase-referenced imaging and micro-arcsecond astrometry facility of the ESO Very Large Telescope Interferometer. The PRIMA Consortium includes Geneva Observatory, Leiden Observatory and Max-Planck Heidelberg. Observations with PRIMA in astrometric mode are planned to start in 2006 with the goal of a final astrometric error of 10 micro-arcseconds per data point. Achieving this accuracy involves a detailed modeling and preparation of the error budget for astrometry observations. In order to reach the above 10 micro-arcsec accuracy, it will be necessary to achieve 50 microns accuracy on the OPD (compared to the millimeter scale that is currently achieved). The global calibration model of PRIMA will be derived from the above error budget and will be defined as a calibration matrix (Fig. 4). The calibration matrix will be adjusted to the observation data using large datasets collected over long periods of time. For PRIMA, the data reduction is expected to be performed at different time scales: per observation, per night and over extended periods of time.

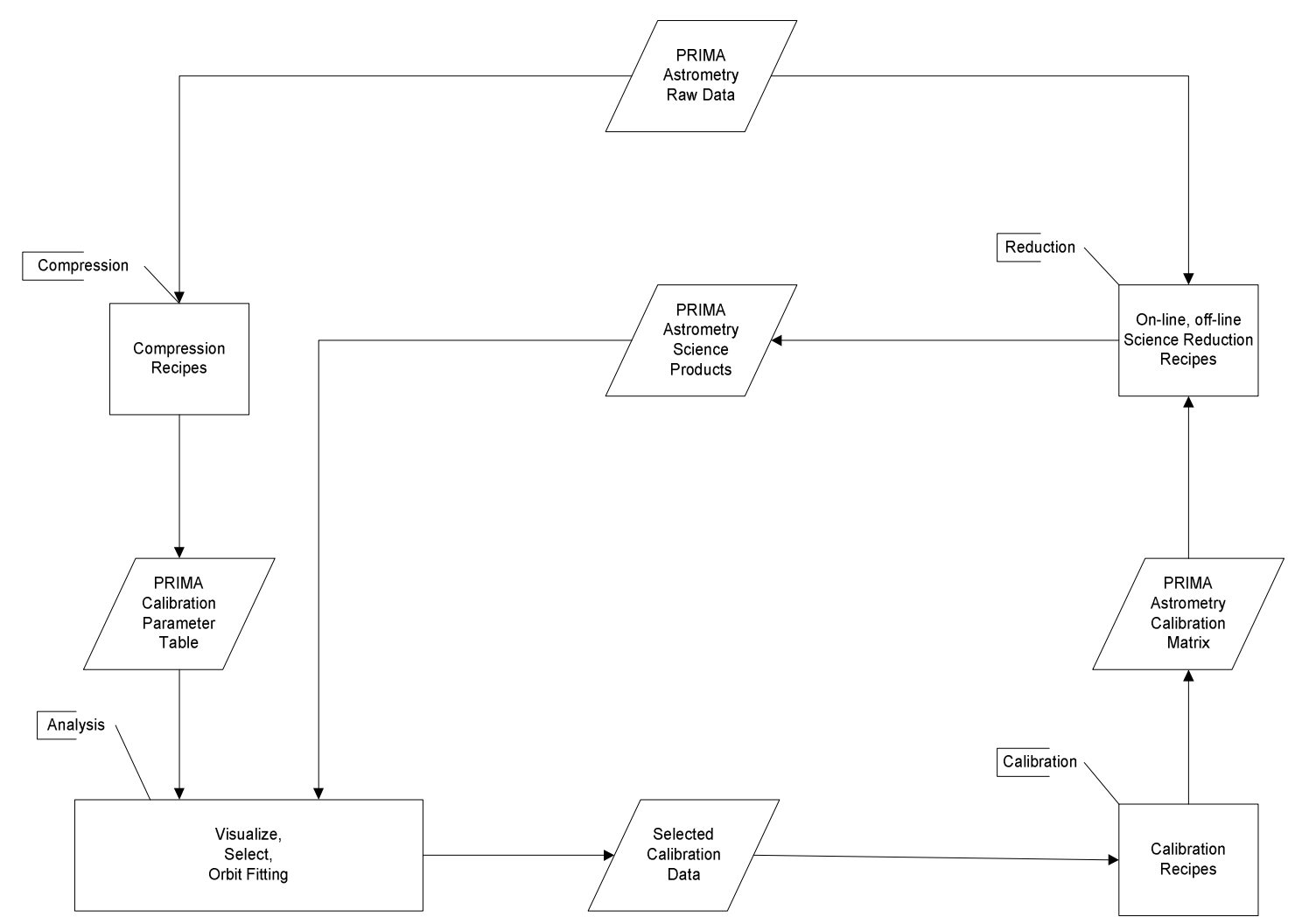

Fig 4. Calibration model for the PRIMA astrometry facility.

\section{CONCLUSION}

The first science instrument MIDI is now integrated into the general data flow and science operations scheme of the VLT. Based on the initial experience with VINCI, specific tools have been developed in the area of observation preparation, data quality control and instrument trending to deal with the specific aspects of interferometry. These developments will remain important as the observational capabilities of the VLTI are extended with the addition of new instruments like AMBER and the Auxiliary Telescopes. The next scientific instrument planned at the VLTI, PRIMA, will rely on fast, homogeneous pipeline processing that allow the quality control scientist to reprocess, if needed, several years of observation data to understand the trends and improve the calibration model of the facility. 


\section{ABBREVIATIONS}

\begin{tabular}{|l|l|}
\hline AMBER & Astronomical Multi-BEam Recombiner \\
AT & Auxiliary Telescope (1.8m) \\
DFS & Data Flow System \\
ETC & Exposure Time Calculator \\
FITS & Flexible Image Transport System \\
FLUOR & Fiber Linked Unit for Optical Recombination \\
mas & milliarcsecond \\
MIDI & Mid-Infrared interferometric instrument \\
OB & Observation Block \\
OPC & Observing Program Committee \\
OPD & Optical Path Difference \\
OPL & Optical Path Length \\
P2PP & Phase 2 Proposal Preparation \\
PRIMA & Phase-Referenced Imaging and Microarcsecond Astrometry \\
QC & Quality Control \\
QC1 & Quality Control Level 1 \\
RAID & Redundant Array of Independent Disks \\
UT & Unit Telescope (8.2m) \\
VINCI & VLT INterferometer Commissionning Instrument \\
VLT & Very Large Telescope \\
VLTI & Very Large Telescope Interferometer \\
\hline
\end{tabular}

\section{REFERENCES}

1. Glindemann, A., et al., 2004, VLTI Technical Advance - Present and Furture, Proc. SPIE 5491-50

2. Jaffe, W., Meisenheimer, K., Röttgering, H. , Leinert, Ch. , Richichi, A., et al., 2004, The central dusty torus in the active nucleus of NGC 1068, Nature, 426, May 6, 2004, p.47

3. Knudstrup, J., et al., 2002, Evolution and Adaptation of the Data Flow System, In Öbservatory Operations to Optimize Scientific Return III, P.J.Quinn, Ed., Proc. SPIE 4844, 213

4. Koehler, B., and the AT1 Assembly and Commissioning team (ESO), 2004, The First Light of the First VLTI Auxiliary Telescope, ESO Messenger, 115, 15

5. Leinert et al., 2003, MIDI Combines Light from the VLTI: the Start of $10 \mu \mathrm{m}$ Interferometry at ESO, ESO Messenger, 112, 13

6. McKay, D.J, et al., 2004, Observation Planning Tools for the VLT Interferometer, 5493-63

7. Morel, S., et al., 2004, Preparing MIDI Science Operations at VLTI, Proc. SPIE 5491-193

8. Pauls, T.A., et al., 2004, A Data Exchange Standard for Optical Interferometry, Proc. SPIE 5491-138

9. Percheron, I., et al., 2004, Quality Control and Instrument Trending for MIDI, Proc. SPIE 5491-134

10. Richichi, A. \& Paresce, F., 2003, Harvesting Scientific Results with the VLTI, ESO Messenger, 114, 26

11. Wittkowski et al., 2004, VLTI/VINCI Observations of the Nucleus of NGC1068 using the Adaptive Optics System MACAO, Astron.Astrophys., 418, 239

12. Wittkowski et al., 2004, Observing with the VLT Interferometer, Proc. SPIE 5491-72 